\title{
Crack Lip Contact Modeling Based on Lagrangian Multipliers with X-FEM
}

\author{
Yuan Jin, Olivier Pierard, Eric Wyart, and Eric Béchet
}

\begin{abstract}
The eXtended Finite Element Method (X-FEM), developed intensively in the past 15 years has become a competitive tool for the solution of problems with evolving discontinuities and singularities. In the present study, we focus on the application of X-FEM on frictionless contact problems in the context of fracture mechanics. A promising approach in the literature counting for this problem consists in applying Lagrangian multipliers. Meanwhile, as pointed out in Ji and Dolbow (Int J Numer Methods Eng 61:2508-2535, 2004), a naive choice for Lagrangian multiplier space leads to oscillatory multipliers on the contact surface. This oscillation results from a non-uniform but mesh-dependent inf-sup condition. In this work, we adapt the algorithm proposed in Béchet et al. (Int J Numer Methods Eng 78:931-954, 2009) on crack lip contact by discretizing the displacement field with both scalar and vector tip enrichment functions (Chevaugeon et al., Int J Multiscale Comput Eng 11:597-631, 2013). The influence of the tip enrichment functions on the stability of the formulation is addressed. We show evidences that the vector enrichment functions can improve the conditioning of the problem without jeopardizing the simulation accuracy in the presence of contact.
\end{abstract}

\section{Introduction}

The eXtended Finite Element Method (X-FEM) [18], which allows for considering crack geometries within elements, is largely used nowadays to handle crack growth problems. This approach, which uses the Partition of Unity [17], enriches the classical basis of shape functions with the so-called enrichment functions to describe the discontinuity of the displacement field across the crack surface.

In crack propagation simulation, the crack lip contact is important to be considered especially when dealing with complex mechanical loadings, e.g. presence

\footnotetext{
Y. Jin • O. Pierard $(\bowtie) \cdot$ E. Wyart

Cenaero, rue des Frère wright 29, 6041 Gosselies, Belgium

e-mail: yuan.jin@cenaero.be; olivier.pierard@cenaero.be; eric.wyart@cenaero.be

E. Béchet

Université de Liège, Allée de la Découverte 9, B-4000 Liège, Belgium

e-mail: eric.bechet@ulg.ac.be
} 
of residual stresses generated during an upstreaming manufacturing process and/or compressive loading. A promising route proposed in the literature to solve contact problem in the X-FEM framework is with Lagrangian multipliers. The idea was firstly introduced by $\mathrm{Ji}$ and Dolbow[14]. The authors report that a naive discretization of the Lagrangian multipliers violates the inf-sup condition, which determines the stability of the formulation. The problem can be fixed by defining a reduced Lagrangian multiplier space as proposed in $[3,8,10,20]$. In these works, only Heaviside functions are introduced to represent the jump across the implicit interface. Nevertheless, for crack lip contact, the tip enrichment functions are necessary for the description of the stress singularity around the crack tip and thus for the calculation of the stress intensity factors (SIFs). In other words, these asymptotic functions cannot be omitted in crack propagation simulations. The algorithm of Lagrangian space construction proposed in [3] was applied to model crack lip contact with classical scalar tip enrichment functions on structured meshes in [25]. Meanwhile, the effect of these functions on the stability of the formulation was not discussed by the authors [25]. The present study aims at modeling frictionless crack lip contact on unstructured meshes. Both Heaviside function and tip enrichment functions are used to discretize the displacement field. The influence of the tip enrichment functions on the stability of the formulation is specifically addressed.

Besides the advantage of relaxing the constraint on finite element mesh, X-FEM is reported to be able to reach the optimal convergence rate with first-order shape functions when the geometrical enrichment strategy is used [2, 15]. However, this enrichment strategy can result in high condition number of the stiffness matrix. The conditioning problem prevents applying this method on complex large industrial problems. Several alternatives are proposed in the literature to address this issue by applying different types of enrichment functions, e.g. the stabilized GFEM [12, 13], the vector enrichment functions $[6,23]$. In this work, in addition to classical scalar tip enrichment functions, vector enrichment functions proposed by Chevaugeon et al. [6] are also applied to model crack lip contact. Their performances in terms of simulation accuracy and conditioning are compared.

The paper is organized as follows. We recall at first the contact formulation. Secondly the discretization of the displacement field and the Lagrangian multipliers is presented. The influence of the discretization on the stability of the formulation is then discussed. The fourth section consists in a description of the SIF calculation in the case of crack lip contact. In the last section of the paper, two two-dimensional test cases are applied to analyze the performance of the proposed strategy.

\section{Formulation of the Continuous Problem}

Hereinafter, bold symbols denote vectors or tensors. We consider a cracked domain $\Omega$ in $\mathbb{R}^{2}$. The two sides of the crack are denoted by $\Gamma_{c+}$ and $\Gamma_{c-}$ respectively (Fig. 1). Only normal (frictionless) contact condition is considered between $\Gamma_{c+}$ 
Fig. 1 A cracked domain

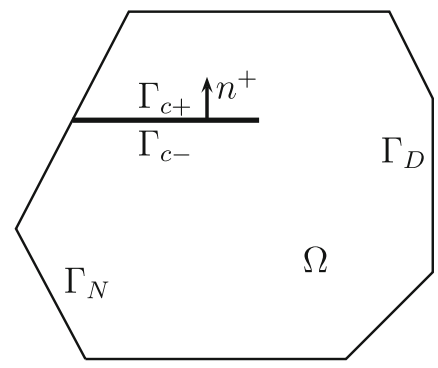

and $\Gamma_{c-}$ in the present study. In the initial configuration, the two sides coincide. $\mathbf{n}\left(=\mathbf{n}^{+}=-\mathbf{n}^{-}\right)$denotes the outward normal unit vector on $\Gamma_{c+}$. The body is subjected to surface loads $\mathbf{g} \in\left(L^{2}\left(\Gamma_{N}\right)\right)^{2}$. Then under planar small strain assumptions, the problem of homogeneous isotropic linear elasticity consists in finding the displacement field $\mathbf{u}: \Omega \rightarrow \mathbb{R}^{2}$ satisfying

$$
\begin{array}{cc}
\nabla \cdot \boldsymbol{\sigma}(\mathbf{u})=0, & \text { in } \Omega, \\
\mathbf{u}=0, & \text { on } \Gamma_{D}, \\
\boldsymbol{\sigma}(\mathbf{u}) \cdot \mathbf{n}=\mathbf{g}, & \text { on } \Gamma_{N},
\end{array}
$$

where $\sigma=\left(\sigma_{i j}\right), 1 \leq i, j \leq 2$, stands for the stress tensor field and '.' is a contraction over a single index. $\sigma$ is defined by the constitutive law for linear elasticity:

$$
\boldsymbol{\sigma}=\mathbf{C}: \boldsymbol{\varepsilon}
$$

where $\mathbf{C}$ is the Hooke tensor, $\boldsymbol{\varepsilon}(\boldsymbol{u})=\left(\nabla \mathbf{u}+\nabla \mathbf{u}^{T}\right) / 2$ represents the linearized strain tensor field and ':'denotes a double contraction.

For a displacement field $\mathbf{u}$, the following notations are adopted to describe the displacement on the contact surface:

$$
\mathbf{u}^{+}=u_{n}^{+} \mathbf{n}^{+}+u_{t}^{+} \mathbf{t}^{+} \quad \text { and } \quad \mathbf{u}^{-}=u_{n}^{-} \mathbf{n}^{-}+u_{t}^{-} \mathbf{t}^{-},
$$

where $\mathbf{t}$ is the unit tangent vector of $\Gamma_{C}$ and $\mathbf{u}^{+}$(resp. $u^{-}$) is the trace of displacement on $\Gamma_{C}^{+}$(resp. on $\Gamma_{C}^{-}$). Then, the frictionless contact condition reads:

$$
\sigma_{n}(\mathbf{u}) \leq 0, \quad \llbracket \mathbf{u} \rrbracket=\left(u_{n}^{+}+u_{n}^{-}\right) \leq 0, \quad \sigma_{n}(\mathbf{u}) \cdot \llbracket \mathbf{u} \rrbracket=0,
$$

where $\sigma_{n}(\mathbf{u})$ is the normal pressure on the contact surface.

In what follows, we adopt classical notations for the functional spaces: $W^{m, p}(\Omega)$ denotes the Sobolev space of functions $v$ for which all components of $m^{\text {th }}$ order weak derivative $D^{m}(v)$ lie in $L^{P}(\Omega)$. The associated norm is denoted by $\|\cdot\|_{m, p, \Omega}$. Following the convention in the literature [8], we note $H^{m}(\Omega):=W^{m, 2}(\Omega)$, for which the index $p=2$ is omitted. The components of the solution to problems 
(1-6) are then belong to the space $\mathbf{V}:=\left\{\mathbf{v} \in\left(H^{1}(\Omega)\right)^{2},\left.\mathbf{v}\right|_{\Gamma_{D}}=0\right\}$. As in [1], we introduce the space:

$$
W=\left\{\left.v_{n}\right|_{\Gamma_{C}}: \mathbf{v} \in \mathbf{V}\right\}
$$

and its dual space $W^{\prime}$. We note $H^{1 / 2}\left(\Gamma_{C}\right)$ the trace space of $H^{1}(\Omega)$ restricted to $\Gamma_{C}$ and $H^{-1 / 2}\left(\Gamma_{C}\right)$ the dual space of $H^{1 / 2}\left(\Gamma_{C}\right)$. Then we have $W \subset H^{1 / 2}\left(\Gamma_{C}\right)$ and $W^{\prime} \subset H^{-1 / 2}\left(\Gamma_{C}\right)$. Let $\lambda:=\mathbf{n}^{T} \cdot \boldsymbol{\sigma} \cdot \mathbf{n}$ be the normal surface force on the contact surface. $\lambda$ then belongs to the space $M=\left\{\lambda \in W^{\prime}:\langle\lambda, \psi\rangle_{W^{\prime}, W} \geq 0\right.$, for all $\psi \in$ $W, \psi \leq 0$ on $\left.\Gamma_{C}\right\}$. The notation $\langle\cdot, \cdot\rangle_{W^{\prime}, W}$ stands for the duality pairing between $W^{\prime}$ and $W$. The weak formulation of the frictionless contact problem then reads, find $\mathbf{u} \in \mathbf{V}$ and $\lambda \in M$ such that

$$
\begin{gathered}
\forall \mathbf{u}^{*} \in \mathbf{V}, \int_{\Omega} \boldsymbol{\sigma}(\mathbf{u}): \boldsymbol{\varepsilon}\left(\mathbf{u}^{*}\right) \mathrm{d} \Omega-\int_{\Gamma_{C}} \lambda \llbracket \mathbf{u}^{*} \rrbracket \mathrm{d} \Gamma=\int_{\Gamma_{N}} \mathbf{g} \cdot \mathbf{u}^{*} \mathrm{~d} \Gamma . \\
\forall \lambda^{*} \in M, \int_{\Gamma_{C}} \lambda^{*} \llbracket \mathbf{u} \rrbracket \mathrm{d} \Gamma=0 .
\end{gathered}
$$

\section{Discretization}

The discretization of the problem (8)-(9) involves a pair of finite element spaces $\mathbf{V}^{h} \subset \mathbf{V}$ and $M^{h} \subset M$ leading to the discrete formulation: find $\left(\mathbf{u}^{h}, \lambda^{h}\right) \in \mathbf{V}^{h} \times M^{h}$ such that

$$
\begin{gathered}
\forall \mathbf{u}^{* h} \in \mathbf{V}^{h}, \int_{\Omega} \boldsymbol{\sigma}\left(\mathbf{u}^{h}\right): \boldsymbol{\varepsilon}\left(\mathbf{u}^{* h}\right) \mathrm{d} \Omega-\int_{\Gamma_{C}} \lambda^{h} \llbracket \mathbf{u}^{* h} \rrbracket \mathrm{d} \Gamma=\int_{\Gamma_{N}} \mathbf{g} \cdot \mathbf{u}^{* h} \mathrm{~d} \Gamma . \\
\forall \lambda^{* h} \in M^{h}, \int_{\Gamma_{C}} \lambda^{* h} \llbracket \mathbf{u}^{h} \rrbracket \mathrm{d} \Gamma=0 .
\end{gathered}
$$

In this work, we suppose that $\Omega$ is meshed with a family of affine triangular meshes $\mathscr{T}_{h}$, which is assumed to be quasi-uniform and regular. The choice of the $\mathbf{V}^{h}$ and $M^{h}$ has to satisfy a uniform inf-sup condition [4] with respect to suitable norms. In the present study, as in $[3,8]$, the following mesh-dependent $L^{2}$ norms are considered:

$$
\begin{aligned}
\left\|\lambda^{h}\right\|_{-1 / 2, \Gamma_{C}}^{2} & :=\sum_{e \in \Gamma_{c}} h_{e}\left\|\lambda^{h}\right\|_{0, e}^{2}, \\
\left\|\mathbf{u}^{h}\right\|_{1 / 2, \Gamma_{C}}^{2} & :=\sum_{e \in \Gamma_{c}} \frac{1}{h_{e}}\left\|\mathbf{u}^{h}\right\|_{0, e}^{2},
\end{aligned}
$$


where $h_{e}$ denotes the length of the segment $e$ on $\Gamma_{C}$. Then in the present context, the inf-sup condition reads:

$$
\exists c<0, \text { independent of } \mathrm{h}, \forall \lambda_{h} \in M^{h}, \sup _{\mathbf{u}^{h} \in \mathbf{V}^{h}} \frac{\int_{\Gamma_{C}} \lambda^{h} \llbracket \mathbf{u}^{h} \rrbracket \mathrm{d} \Gamma}{\left\|\mathbf{u}^{h}\right\|_{1, \Omega}} \geq c h^{1 / 2}\left\|\lambda^{h}\right\|_{0, \Gamma_{C}}
$$

where $h:=\max _{e} h_{e}$.

Let $U_{h}$ and $L_{h}$ be respectively the vector of the displacement and the Lagrangian multipliers. Then the algebraic form of the problem (10)-(11) reads:

$$
\left(\begin{array}{cc}
\mathbf{A}_{h} & \mathbf{B}_{h}^{T} \\
\mathbf{B}_{h} & 0
\end{array}\right)\left(\begin{array}{c}
U_{h} \\
L_{h}
\end{array}\right)=\left(\begin{array}{c}
F_{h} \\
0
\end{array}\right)
$$

To evaluate numerically the inf-sup condition, thanks to the work in $[3,5]$, the following algebraic equation is introduced:

$$
\left(\begin{array}{cc}
\mathbf{A}_{h} & \mathbf{B}_{h}^{T} \\
\mathbf{B}_{h} & \frac{1}{k} \mathbf{M}_{h}
\end{array}\right)\left(\begin{array}{c}
U_{h} \\
L_{h}
\end{array}\right)=\left(\begin{array}{c}
F_{h} \\
0
\end{array}\right)
$$

where $\mathbf{M}_{h}$, a symmetric mass matrix, corresponds to the integral on $\Gamma_{C}$ $\left(\int_{\Gamma_{C}} \lambda^{h} \lambda^{* h} \mathrm{~d} \Gamma\right.$ ). Equation (16) is equivalent to Eq. (15) when $k$ tends to infinity. We denote $\beta_{h ; \text { min }}$ the first non-zero eigenvalue of the following problem:

$$
\frac{1}{h}\left(\mathbf{B}_{h}^{T} \mathbf{M}_{h}^{-1} \mathbf{B}_{h}\right) V_{h}=\beta_{h} \mathbf{A}_{h} V_{h}
$$

Then the value of $c$ in Eq. (14) is approximated by $\min _{h_{i}} \beta_{h ; m i n}^{0.5}$.

\subsection{Discretization of the Displacement Field}

Let $\mathscr{E}_{h}$ be the set of elements of $\mathscr{T}_{h}$ completely cut by $\Gamma_{C}$ and $\mathscr{V}_{h}$ the corresponding intersected edges. We denote $\mathscr{N}_{h} \subset \mathbb{N}$ the nodes of the mesh, $\mathscr{K}_{h} \subset \mathscr{N}_{h}$ the nodes of elements in $\mathscr{E}_{h}$. X-FEM is applied to count for the displacement jump across the crack surface. The discretized displacement field is expressed as follows:

$$
\mathbf{u}^{h}=\sum_{\mathscr{N}_{h}} N_{i}(\mathbf{x}) \mathbf{u}_{i}+\sum_{\mathscr{K}_{h}} N_{k}(\mathbf{x}) H(\mathbf{x}) \mathbf{a}_{k} .
$$

The Heaviside-like step function $H(\mathbf{x})$, with a changing sign across the crack, enriches the nodes in $\mathscr{K}_{h}$. Classically, it is defined thanks to the signed distance function to the crack $\phi$, i.e. the so-called normal level set (see [19, 26]):

$$
H(\mathbf{x})=\operatorname{sign}(\phi(\mathbf{x})) .
$$


In addition to Heavisde functions, tip enrichment functions are also applied on the set of nodes within a prescribed enrichment radius to the crack tip $\mathscr{J}_{h} \subset \mathscr{N}_{h}$ for the purpose of describing the stress singularity. In the present study, two types of tip enrichment functions denoted respectively by scalar enrichment functions and vector enrichment functions are used. For classical scalar enrichment functions, the displacement field is approximated by

$$
\mathbf{u}^{h}=\sum_{\mathscr{N}_{h}} N_{i}(\mathbf{x}) \mathbf{u}_{i}+\sum_{\mathscr{K}_{h}} N_{k}(\mathbf{x}) H(\mathbf{x}) \mathbf{a}_{k}+\sum_{\mathscr{J}_{h}} N_{j}(\mathbf{x})\left(\sum_{l} f_{l}(\mathbf{x}) \mathbf{b}_{k l}\right),
$$

where

$$
f=[\sqrt{r} \cos (\theta / 2), \sqrt{r} \sin (\theta / 2), \sqrt{r} \sin (\theta / 2) \sin \theta, \sqrt{r} \cos (\theta / 2) \sin (\theta)] .
$$

$(r, \theta)$ represents the polar coordinate system associated with the crack tip. The vector enrichment functions are introduced in [6]:

$$
\mathbf{u}^{h}=\sum_{\mathscr{N}_{h}} N_{i}(\mathbf{x}) \mathbf{u}_{i}+\sum_{\mathscr{K}_{h}} N_{k}(\mathbf{x}) H(\mathbf{x}) \mathbf{a}_{k}+\sum_{\mathscr{J}_{h}} \sum_{\alpha} N_{j}(\mathbf{x}) \mathbf{K}_{\alpha}(\mathbf{x}) c_{j \alpha},
$$

where

$$
\begin{gathered}
\mathbf{K}_{1}=\sqrt{r} \cos (\theta / 2)(k-\cos (\theta))\left[\mathbf{e}_{1}(\mathbf{x})+\mathbf{e}_{2}(\mathbf{x})\right], \\
\mathbf{K}_{2}=\sqrt{r} \sin (\theta / 2)(k+2+\cos (\theta)) \mathbf{e}_{1}(\mathbf{x})+\sqrt{r} \cos (\theta / 2)(k-2 \cos (\theta)) \mathbf{e}_{2}(\mathbf{x}),
\end{gathered}
$$$$
\mathbf{K}_{3}=\sqrt{r} \sin (\theta / 2) \mathbf{e}_{3}(\mathbf{x}) .
$$

As indicated in Eqs. (23)-(25), $\mathbf{K}_{\alpha}, \alpha \in\{1,2,3\}$ are defined on the local basis $\mathbf{e}_{i}, i \in$ $\{1,2,3\}$. The vector enrichment functions limit the number of degrees of freedom per enriched node to 3 (to be compared to the 12 degrees of freedom per node with scalar enrichment functions). More details of these two types of enrichment functions are provided in $[2,6]$.

\subsection{Discretization of the Lagrangian Multipliers}

As in $[8,10]$, the Lagrangian multiplier components are defined on the parent nodes belonging to $\mathscr{K}_{h}$. They are based on the same linear shape functions $N_{i}(\mathbf{x})$ that are used for the displacement field. The approximation space $M^{h}$ for the multiplier is then:

$$
M^{h}=\left\{\left.\lambda^{h}\right|_{\Gamma}, \lambda^{h} \in M^{\prime h}\right\}
$$


where

$$
M^{\prime h}:=\left\{\mathbf{x} \in \mathscr{E}_{h} \rightarrow \sum_{i \in \mathscr{K}_{h}} \lambda_{i} N_{i}(\mathbf{x}), \lambda_{i} \in \mathbb{R}\right\}
$$

With X-FEM, a naive linear $P 1-P 1$ interpolation choice for the displacement and Lagrangian multipliers respectively is not stable and results in oscillations [14]. An adequate reduction of the Lagrangian multiplier space can efficiently address the problem [3, 8]. In the algorithm proposed by Béchet et al. [3], a piecewise linear or constant interpolation is adopted for the Lagrangian multipliers. This interpolation, which can be considered as between $P 1$ and $P 0$, is denoted by $P 1^{-}$in the present study. Accordingly, combined with the linear interpolation of the displacement, the formulation used is denoted by $P 1 / \mathrm{P1}^{-}$. Another approach was proposed by Ferté et al. [8]. By releasing some constraint from $P 1^{-}$, the new algorithm of $P 1$ multipliers, referred to $P 1^{*}$, verifies $P 1^{-} \subset P 1^{*}$. The formulation is combined with a piecewise quadratic displacement, which is denoted by $P 2 / P 1^{*}$. Both approaches are proven mathematically to be able to satisfy the inf-sup condition. Roughly speaking, with the constructed Lagrangian multiplier space, it is possible to find $\mathbf{w}^{h} \in \mathbf{V}^{h}$ that $\lambda^{h}=\llbracket \mathbf{w}^{h} \rrbracket$. Then it is sufficient to show $\frac{\int_{\Gamma_{C}} \lambda^{h} \llbracket \mathbf{w}^{h} \rrbracket \mathrm{d} \Gamma}{\left\|\mathbf{w}^{h /}\right\|_{1, \Omega}} \geq c h^{1 / 2} \|$ $\lambda^{h} \|_{0, \Gamma_{C}}$. We refer to $[3,8]$ for more details of this demonstration.

In this work, a linear interpolation on the displacement field is adopted. Accordingly, the algorithm of Béchet is applied to construct the Lagrangian multiplier space. It is noteworthy that in [3, 8], the implicit interface completely crosses the simulation domain. However, for contact between crack lips, the contact interface ends within the simulation domain. Therefore, a special treatment is applied on the element containing the crack tip. As illustrated in Fig. 2, the element with nodes $q_{i}^{c t}, i \in 1,2,3$ contains the crack tip. If the edge $q_{1}^{c t}-q_{3}^{c t}$ supports an equality relationship, then a complementary equality relationship is imposed so that the degree of freedom of the Lagrangian multiplier defined on $q_{2}^{c t}$ equals the ones on $q_{1}^{c t}$ and $q_{3}^{c t}$. Otherwise, since either $q_{3}^{c t}$ or $q_{1}^{c t}$ is connected to another edge supporting an equality relationship (see [3]), a single equality relationship is imposed among

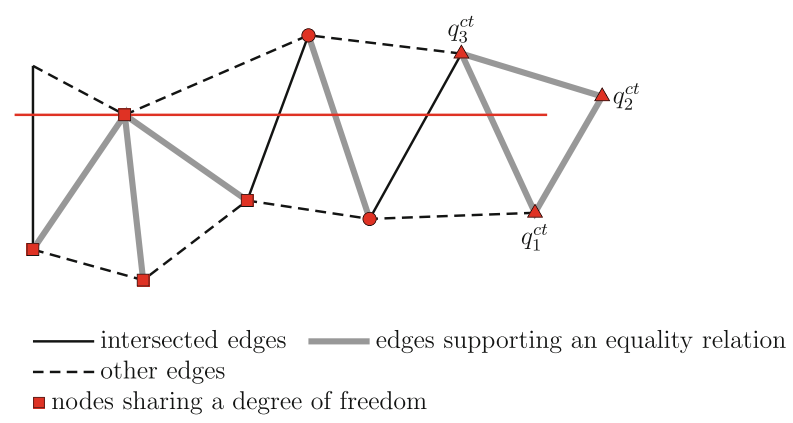

Fig. 2 Restriction algorithm $P 1^{-}$defined by Béchet et al. [3] 
$q_{i}^{c t}, i \in 1,2,3$ so that the node connects the other two nodes. In summary, a $P 0$ interpolation is used in this element for Lagrangian multipliers.

Furthermore, in the work of Béchet et al. [3], only the Heavisde function is used for enrichment. In the present study, the tip enrichment functions given in Eqs. (21) and (23)-(25) are also applied to describe the stress singularity around the crack tip. The application of these functions does not violate the inf-sup condition. In fact, by simply imposing the related degrees of freedom ( $\mathbf{b}_{k l}$ in Eq. (20) or $c_{j \alpha}$ in Eq. (22)) equal to zero, we can easily find a displacement field $\mathbf{w}^{h} \in \mathbf{V}^{h}$ that satisfies $\lambda^{h}=\llbracket \mathbf{w}^{h} \rrbracket$. Then the demonstration procedure proposed in [3] proves that with this displacement discretization the inf-sup condition is respected.

\section{Stress Intensity Factors}

An accurate calculation of the stress intensity factors (SIFs) is essential for crack propagation simulation. In the present study, the SIFs are extracted from the XFEM solution thanks to the interaction integrals in a domain form [7, 16, 24, 28]. Here, we recall the calculation procedure. For this, two states of a cracked body are defined:

- state $1=$ present state $\left(u_{i}^{1}, \sigma_{i j}^{1}, \varepsilon_{i j}^{1}\right)$,

- state $2=$ auxiliary state $\left(u_{i}^{2}, \sigma_{i j}^{2}, \varepsilon_{i j}^{2}\right)$.

Accordingly, the interaction integral $I^{(1,2)}$ can be calculated as follows:

$$
I^{(1,2)}=\oint_{\Gamma}\left[W^{(1,2)} \delta_{1 j}-\sigma_{i j}^{1} \frac{\partial u_{i}^{2}}{\partial x_{1}}-\sigma_{i j}^{2} \frac{\partial u_{i}^{1}}{\partial x_{1}}\right] n_{j} \mathrm{~d} \Gamma,
$$

where $\delta$ is the Kronecker's delta and $W^{(1,2)}$ is the interaction strain energy defined as

$$
W^{(1,2)}=\sigma_{i j}^{(1)} \varepsilon_{i j}^{(2)}=\sigma_{i j}^{(2)} \varepsilon_{i j}^{(1)} .
$$

The direction $x_{1}$ is defined by the local basis associated with the crack tip (see Fig. 3). By defining a weighting function $q(x)$ :

$$
q= \begin{cases}1 & \text { on } \Gamma, \\ 0 & \text { on } C_{0}, \\ \text { arbitrary } & \text { elsewhere, }\end{cases}
$$


Fig. 3 Contour integral around the crack tip

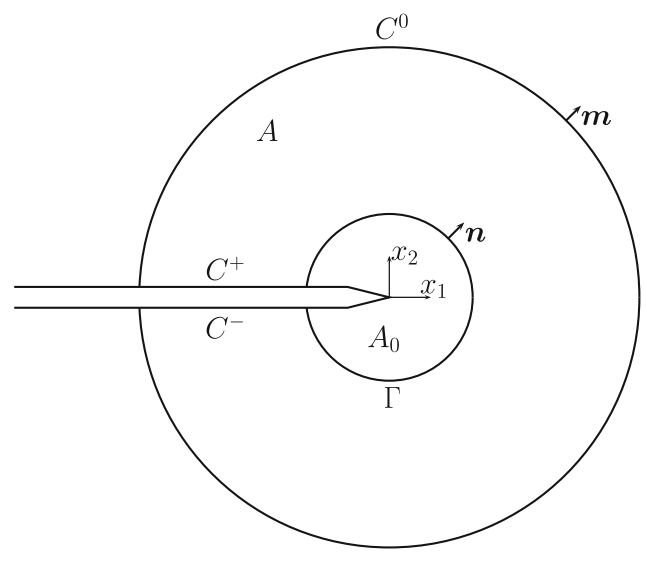

Equation (28) can be reformulated as:

$$
\begin{gathered}
I^{(1,2)}=\int_{C}\left[-W^{(1,2)} \delta_{1 j}+\sigma_{i j}^{1} \frac{\partial u_{i}^{2}}{\partial x_{1}}+\sigma_{i j}^{2} \frac{\partial u_{i}^{1}}{\partial x_{1}}\right] q m_{j} \mathrm{~d} C \\
-\int_{C_{+}+C_{-}}\left[-W^{(1,2)} \delta_{1 j}+\sigma_{i j}^{1} \frac{\partial u_{i}^{2}}{\partial x_{1}}+\sigma_{i j}^{2} \frac{\partial u_{i}^{1}}{\partial x_{1}}\right] q m_{j} \mathrm{~d} C,
\end{gathered}
$$

where $C$ is the contour of the Equivalent Domain integral, i.e.

$$
C=\Gamma+C_{+}+C_{-}+C_{0}
$$

and $\mathbf{m}$ is the unit outward normal of $C$ as illustrated in Fig. 3. On $\Gamma$, we have $\mathbf{m}=-\mathbf{n}$.

$I^{(1,2)}$ can be written for a general mixed modes (I-II-III) fracture [27]:

$$
I^{(1,2)}=\frac{2}{E^{*}}\left(K_{I}^{1} K_{I}^{2}+K_{I I}^{1} K_{I I}^{2}\right)+\frac{K_{I I}^{1} K_{I I I}^{2}}{\mu},
$$

where $E^{*}=\frac{E}{1-v^{2}}$ in the present study (plane strain). $E$ and $v$ denote respectively the Young's modulus and the Poisson ratio. To determine $K_{I}^{1}$, the auxiliary field can be chosen as the asymptotic solution of a pure mode I state so that $K_{I}^{2}=1, K_{I I}^{2}=0$ and $K_{I I I}^{2}=0$. Then $K_{I}^{1}$ can be deduced from Eq. (33) as follows:

$$
K_{I}^{1}=\frac{E^{*}}{2 K_{I}^{2}} I^{(1,2)}=\frac{E^{*}}{2} I^{(1,2)} .
$$

For such auxiliary field, $\sigma_{i j}^{(2)}=0$ for $i=1,2$ and $j=1,2$ on $C_{+}$and $C_{-}$. Accordingly, in case of crack lip contact, the interaction integral $I^{(1,2)}$ can be 
written as

$$
\begin{aligned}
I^{(1,2)}=\int_{A}\left[-W^{(1,2)} \delta_{1 j}\right. & \left.+\sigma_{i j}^{(1)} \frac{\partial u_{i}^{(2)}}{\partial x_{1}}+\sigma_{i j}^{(2)} \frac{\partial u_{i}^{(1)}}{\partial x_{1}}\right] \frac{\partial q}{\partial x_{j}} \mathrm{~d} A \\
& -\int_{C_{+}+C_{-}}\left[\sigma_{i j}^{(1)} \frac{\partial u_{i}^{(2)}}{\partial x_{1}}\right] q m_{j} \mathrm{~d} C .
\end{aligned}
$$

It is worth emphasizing that different from traction-free cracks, in the case of crack lip contact, the second term in Eq. (35) cannot be ignored. Details of the calculation procedure of the SIFs can be found in [21,22].

\section{Numerical Experiments}

In this section, two two-dimensional (2D) frictionless contact problems are used to illustrate the performance of the algorithm described in the previous section. In these two cases, contact forces along the crack are respectively uniform and non-uniform compression. The numerical solutions are compared with analytical values of the Lagrangian multipliers. Plane strain conditions are assumed in both examples. The Young's modulus and the Poisson ratio and are taken respectively as equal to 1 and 0.3 .

\subsection{Cracked Block Under Uniform Pressure}

A uniform pressure (0.01) is imposed on the top surface of a square sample [2 $\times 2]$, whereas the bottom right corner is restrained in both directions and the bottom left corner in the vertical $(y)$ one. A crack is inserted into the square sample at $y=0$ (from $x=-1$ to $x=-0.6$ ). The test case is illustrated in Fig. 4.

Fig. 4 Uniform pressure imposed on the top surface of a 2D square sample with a crack (red line) inside

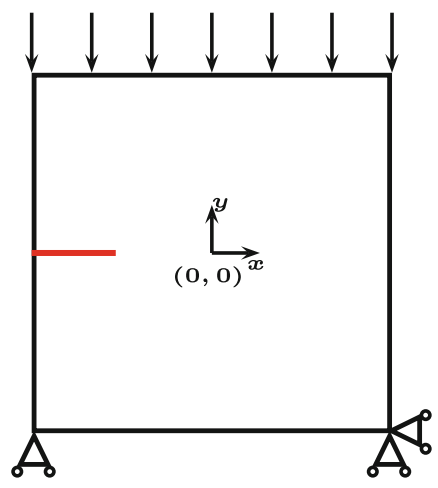


(a)

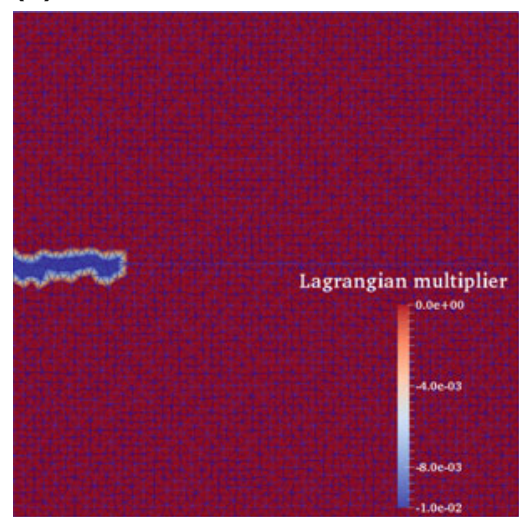

(b)

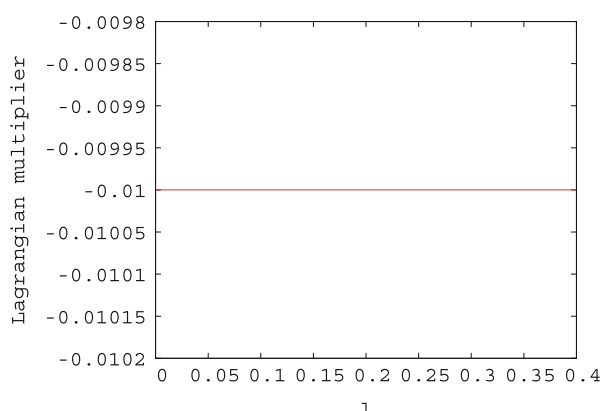

Fig. 5 2D compression test case; displacement field is only enriched with Heaviside functions; (a) Lagrangian multiplier in the simulation domain; (b) the value of the Lagrangian multiplier on the crack expressed as a function of the distance to the left extremity of the crack

To solve the above problem, firstly as in [3] only Heaviside functions are used as enrichment functions to discretize the displacement field. The problem is solved on an unstructured mesh. 42 equal subdivisions are used along each edge of the square. The mesh size $h$ is thus about 0.05 . Applying the previously presented algorithm, the resulted Lagrangian multiplier field is illustrated in Fig. 5. With an adequate Lagrangian multiplier space, the Lagrangian multiplier is constant along the crack and the value corresponds to the pressure imposed on top surface.

Now, for the same problem, the two types of tip enrichment functions are included in the displacement field discretization as indicated by Eqs. (21) and (23)(25). Geometrical enrichment strategy is applied and the enrichment radius of the tip enrichment functions equals 0.2. However, the application of the full description of the displacement field leads to an oscillation of the Lagrangian multiplier along the crack as illustrated in Fig. 6a. As the same results in terms of Lagrangian multipliers are obtained with the vector enrichment functions, only the results of the scalar functions are shown in this section.

The magnitude of the oscillation is around $1 \%$. Meanwhile it can be limited to the crack tip by refining the mesh (Fig. 6b). This problem is thus not related to the instability of the formulation. The numerical inf-sup test (Eq. (16)) is performed on this test case. The result is given in Fig. 7. The inf-sup value with a naive Lagrangian multiplier space (with only Heavisde functions) tends to zero, which indicates a violation of the inf-sup condition. On the other hand, with the adequate discretization, regardless the type of tip enrichment functions, the value is more or less constant as the mesh is refined. This observation further shows that the inf-sup condition is respected with tip enrichment functions. The oscillation is very likely to be a numerical error of the finite element resolution. 
(a)

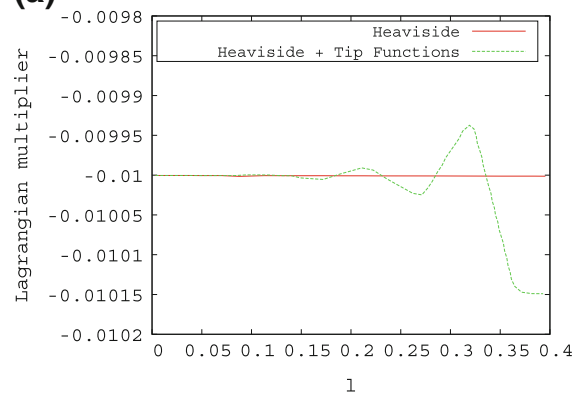

(b)

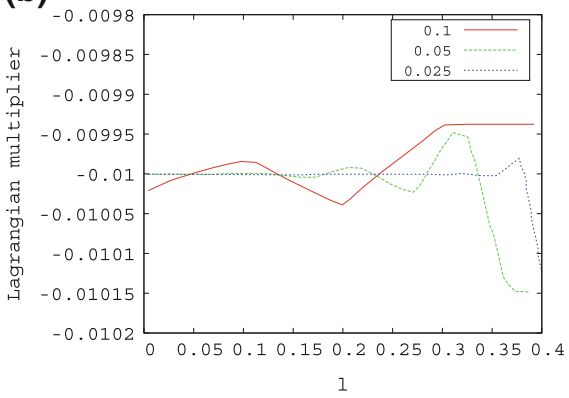

Fig. 6 Oscillation of the Lagrangian multiplier around the crack tip using the scalar tip enrichment functions; (a) result on the mesh shown in Fig. 5; (b) comparison of the results on different meshes(the mesh size $h$ is indicated in the Figure)

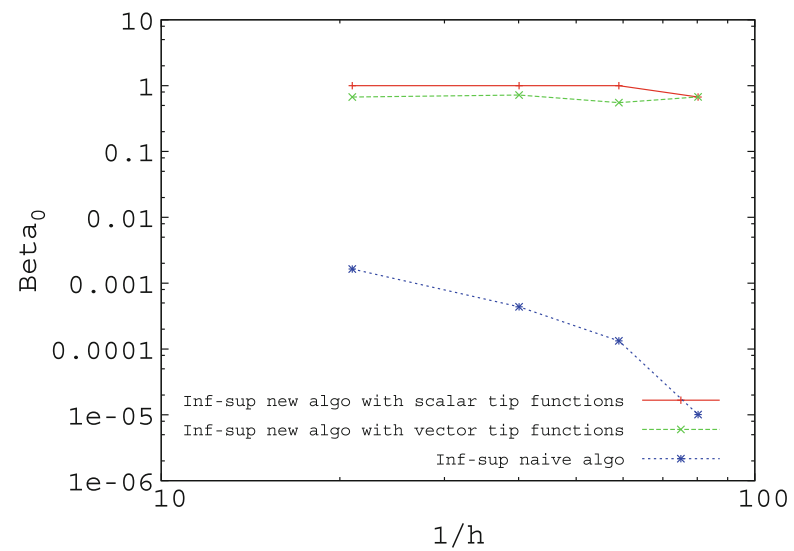

Fig. 7 Numerically computed inf-sup value

Up to now, the integration degree (IntDeg) is set to be 5 on the elements within the enrichment radius. In the present numerical context, the number integration points used for the triangular elements equals [IntDeg +3$) / 2]^{2}$ while the number of integration points for the linear elements on the crack surface equals $[$ IntDeg +1$) / 2]([x]$ refers to the integer part of $x)$. The calculation of the stiffness matrix ( $\mathbf{A}_{h}$ in Eq. (15)) contains the integral of the differential of the shape function $f_{l}$, which leads to the integral of the singular functions containing a combination of $\left\{\frac{1}{r}, \frac{1}{\sqrt{r}}, 1, \sqrt{r}, r\right\}$ multiplied by harmonic functions. Therefore, more integration points are needed to improve the calculation accuracy. As shown in Fig. 8, by doing so, we can considerably decrease the magnitude of the Lagrangian multiplier oscillation around the crack tip. 


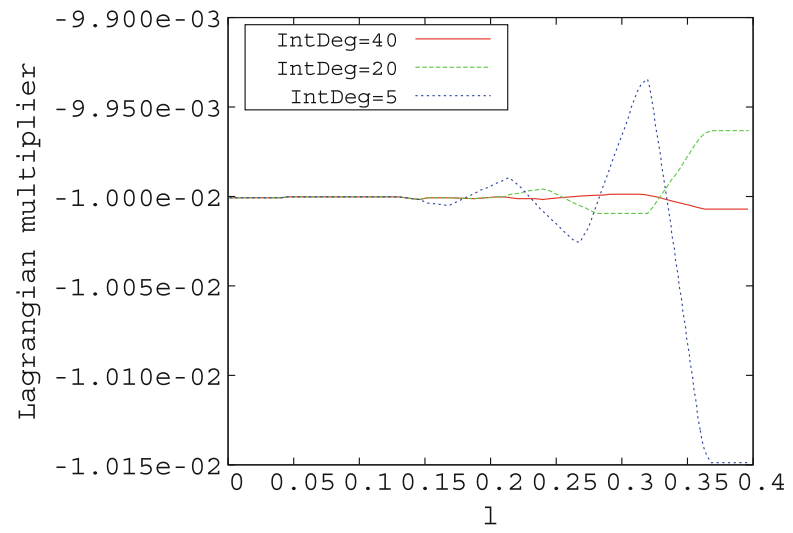

Fig. 8 Lagrangian multiplier value along the crack surface using different integration degrees

Besides the Lagrangian multipliers, the calculation of the SIFs is also analyzed. Since it is a two-dimensional compressive case and only the normal contact is considered, mode I is predominant with respect to mode II. Accordingly, we focus on $K_{I}$ in this analysis. The discontinuity should be completely removed when the contact condition is fully respected. The theoretical solution of $K_{I}$ in this case is thus zero. As the local basis is aligned with the global basis and the normal to the crack surface is the axis $y$, by making use of the asymptotic solution of pure opening mode I as the auxiliary field, Eq. (35) can be further simplified as

$$
\begin{aligned}
I^{(1,2)}=\int_{A}\left[-W^{(1,2)} \delta_{1 j}\right. & \left.+\sigma_{i j}^{(1)} \frac{\partial u_{i}^{(2)}}{\partial x_{1}}+\sigma_{i j}^{(2)} \frac{\partial u_{i}^{(1)}}{\partial x_{1}}\right] \frac{\partial q}{\partial x_{j}} \mathrm{~d} A \\
& +\int_{C_{+}+C_{-}}\left[\lambda \frac{1}{4 \mu} \frac{k+1}{\sqrt{2 \pi r}}\right] q \mathrm{~d} C,
\end{aligned}
$$

where $k=3-4 v$ under the plane strain assumption. Therefore, the accuracy of the SIF calculation depends on the integral of the singular function $1 / \sqrt{r}$. The absolute errors in $K_{I}$ obtained by applying different integration degrees are expressed as a function of mesh size $(h)$ in Fig. 9. In the present numerical context, for the integral on the crack surface, the crack tip is considered as a node of the surface mesh. Then the relative position of the crack tip with respect to the finite element containing it can have an influence on the integral of the singular function $\frac{1}{\sqrt{r}}$. Here to simplify the discussion, the analysis shown in Fig. 9 is performed exceptionally on structured meshes, which do not fit the crack surface.

As expected, the error in $K_{I}$ decreases as more integration points and/or smaller elements are used. As indicated in Fig. 9, the convergence rate equals 0.5 , which corresponds to the one of the integral of $1 / \sqrt{r}$. This observation shows for this problem since the calculation of the Lagrangian multiplier is rather accurate especially when 
Fig. 9 Evolution of the error in $K_{I}$ obtained with different integration degrees as a function of mesh size

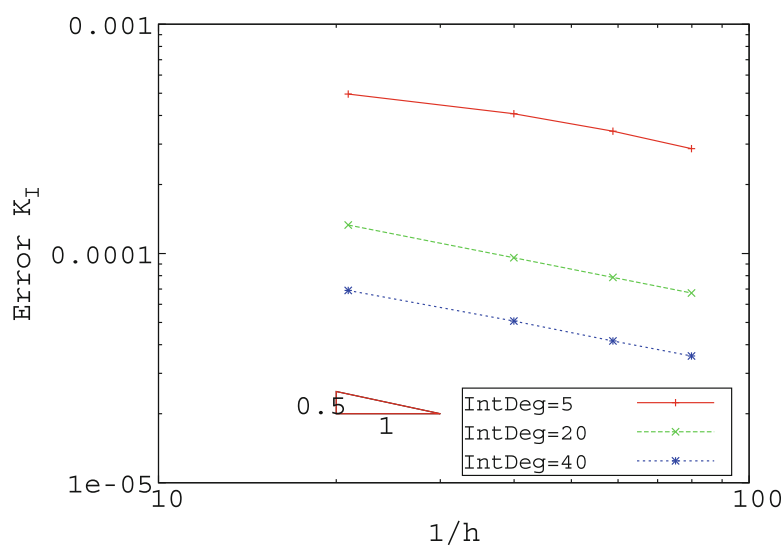

an elevated degree of integration is used (see Fig. 8), the convergence rate of $K_{I}$ is mostly determined by the integration accuracy of the singular function $1 / \sqrt{r}$ on $C_{+}$ and $C_{-}$. Addressing this point with a more adapted integration method, e.g. [2, 9] will be a short-term perspective of the present study.

\subsection{Cracked Block Under Non-Uniform Pressure}

In this section, to further test the performance of the proposed algorithm in a more general case, the problem of a crack emanating from a stress raiser is examined: a circular hole in an infinite plate (illustrated in Fig. 10). The exact solution of this problem, given in [11], is expressed in polar coordinates as follows:

$$
\begin{gathered}
\sigma_{r r}(r, \theta)=\frac{\sigma_{\infty}}{2}\left(1-\frac{R^{2}}{r^{2}}\right)+\frac{\sigma_{\infty}}{2}\left(1+\frac{3 R^{4}}{r^{4}}-\frac{4 R^{2}}{r^{2}}\right) \cos 2 \theta, \\
\sigma_{\theta \theta}(r, \theta)=\frac{\sigma_{\infty}}{2}\left(1+\frac{R^{2}}{r^{2}}\right)-\frac{\sigma_{\infty}}{2}\left(1+\frac{3 R^{4}}{r^{4}}\right) \cos 2 \theta, \\
\sigma_{r \theta}(r, \theta)=-\frac{\sigma_{\infty}}{2}\left(1-\frac{3 R^{4}}{r^{4}}+\frac{2 R^{2}}{r^{2}}\right) \sin 2 \theta,
\end{gathered}
$$

where $R$ is the hole radius, $\sigma_{\infty}$ is the remote compressive load and $a$ is the crack length. The problem is solved on a sequence of uniformly refined unstructured meshes.

To facilitate the implementation of the problem, the exact stress is imposed as Neumann boundary conditions on a semi-circular plate as illustrated by the dash line in Fig. 10. The radius of the hole $(R)$ equals 1 , while the radius of the circular 


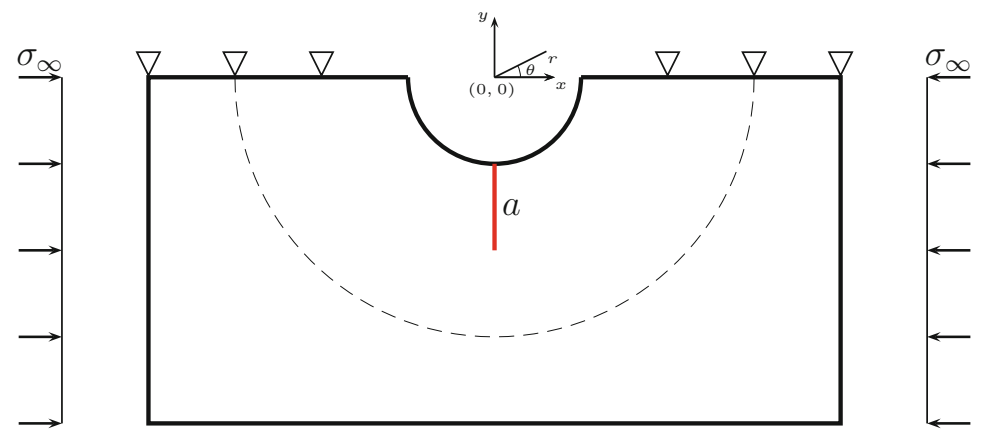

Fig. 10 Uniform pressure imposed on the two sides of an infinite plate with a crack emanating form a circular hole

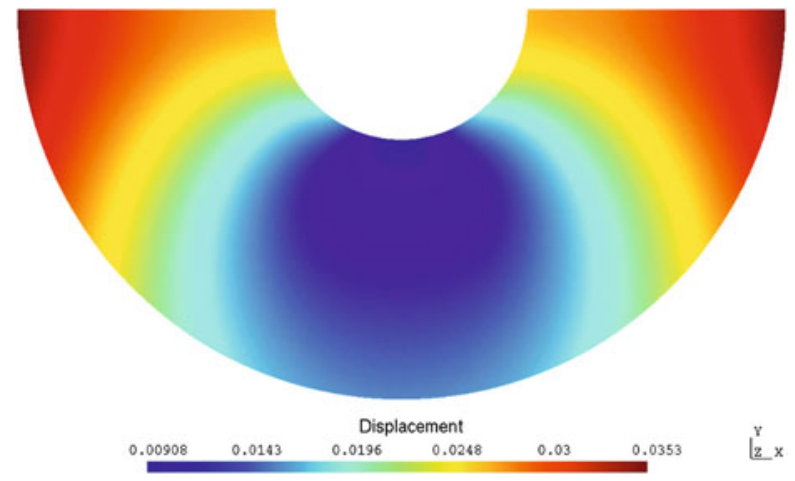

Fig. 11 Solution of the displacement of the infinite plate problem

plate equals 3 . The crack length $(a)$ and $\sigma_{\infty}$ are respectively set to be 1 and 0.01 . Both Heaviside and tip enrichment functions are used to discretize the displacement field. The enrichment radius equals 0.3 . To guarantee the accuracy of the Lagrangian multiplier calculation, the degree of integration is set be 20 for the elements within the enrichment radius. The solution of the displacement field is described in Fig. 11.

The evolution of the energy norm error, which is defined by Eq. (40), is described in Fig. 12.

$$
\operatorname{Err}=\left(\int_{\Omega}\left(\sigma^{e x}-\sigma^{h}\right)^{T} \mathbf{D}\left(\sigma^{e x}-\sigma^{h}\right) \mathrm{d} \Omega\right)^{0.5} .
$$

In addition to the cracked infinite plate problem, a continuous problem without crack in the same configuration has also been solved. With both types of tip enrichment functions, the optimal convergence rate, which is conformed with the continuous problem, is reached. 


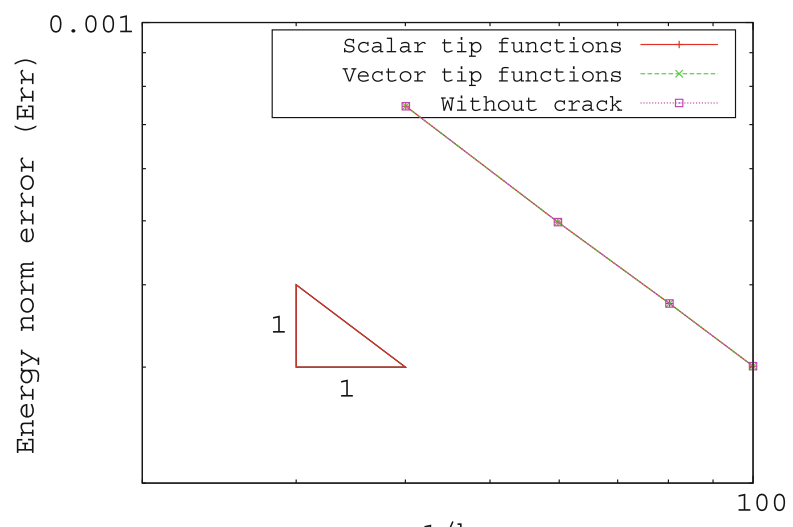

$1 / \mathrm{h}$

Fig. 12 Evolution of the energy norm error of the infinite plate problem

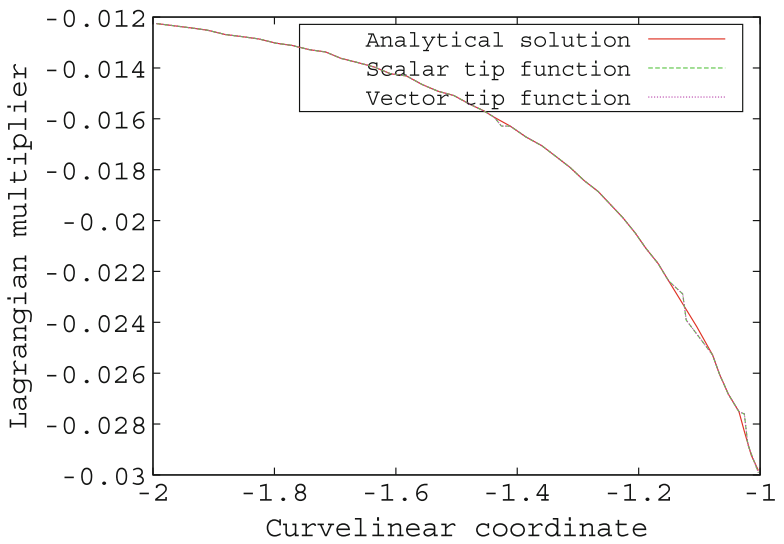

Fig. 13 Profile of the Lagrangian multiplier along the crack obtained on an unstructured mesh $(h=0.025)$ compared with the exact solution

The profile of the Lagrangian multiplier along the crack is compared with the exact solution in Fig. 13. Once again, the numerical solutions with the two types of tip enrichment functions overlap the exact solution. Since the exact stress field is not uniform, to evaluate the calculation of the Lagrangian multiplier, the following $L_{2}$ error is introduced:

$$
\left\|e_{\lambda}\right\|_{L_{2}}=\left[\int_{\Gamma_{C}}\left(\lambda_{e x}-\lambda_{h}\right)^{2} \mathrm{~d} \Gamma\right]^{1 / 2}
$$


Fig. 14 Evolution of the $L_{2}$ error of the Lagrangian multiplier as a function of mesh size $(h)$

Fig. 15 The error in $K_{I}$ in the infinite plate problem expressed as a function of mesh size $(h)$
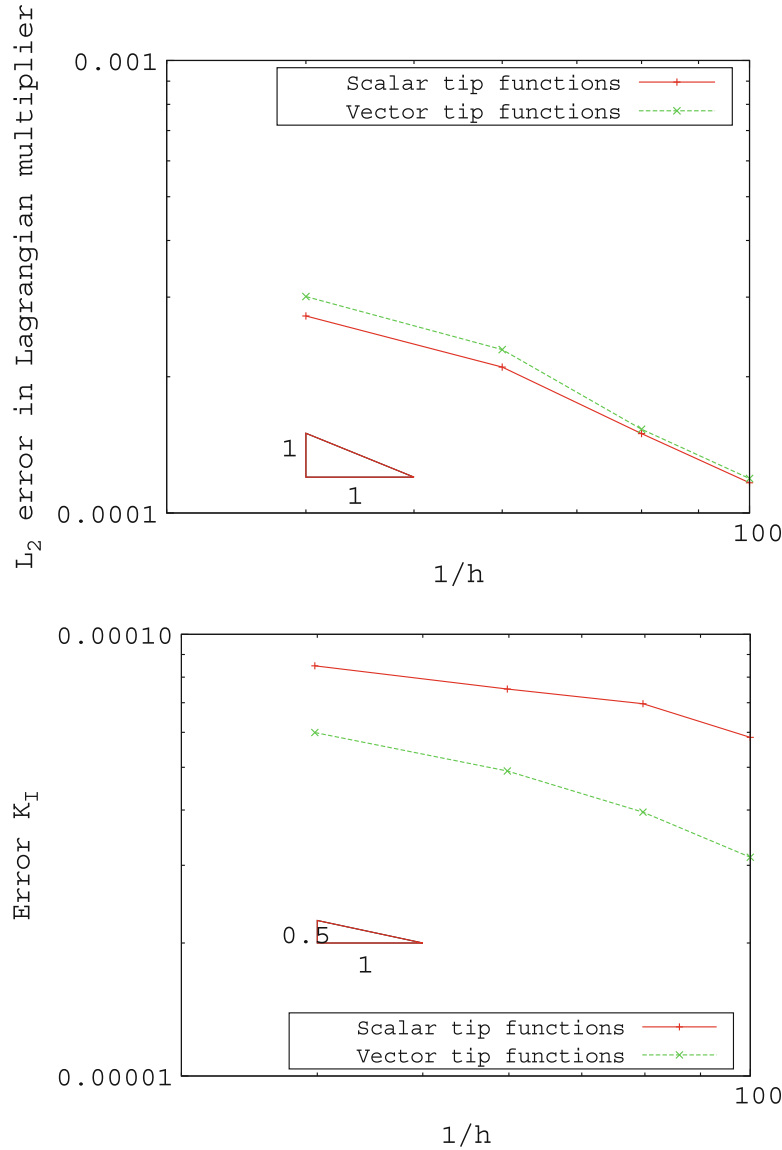

The evolution of the $L_{2}$ error as function of the mesh is described in Fig. 14. As the energy norm error, the errors in the Lagrangian multiplier converge also at the optimal rate, which is equal to 1 .

As in the first test case, mode $\mathrm{I}$ is predominant with respect to the other mode in the infinite plate problem. The absolute error in $K_{I}$ is expressed as a function of mesh size in Fig. 15. For this case, $K_{I}$ is calculated on the unstructured mesh and it does not converge uniformly. Meanwhile, with the two types of enrichment functions, the decrease rate of the absolute error is around 0.5 , which shows despite the non-uniform value of the Lagrangian multipliers, the error in $K_{I}$ is mostly under the influence of the integral accuracy of the singular function $1 / \sqrt{r}$ on the crack surface. 


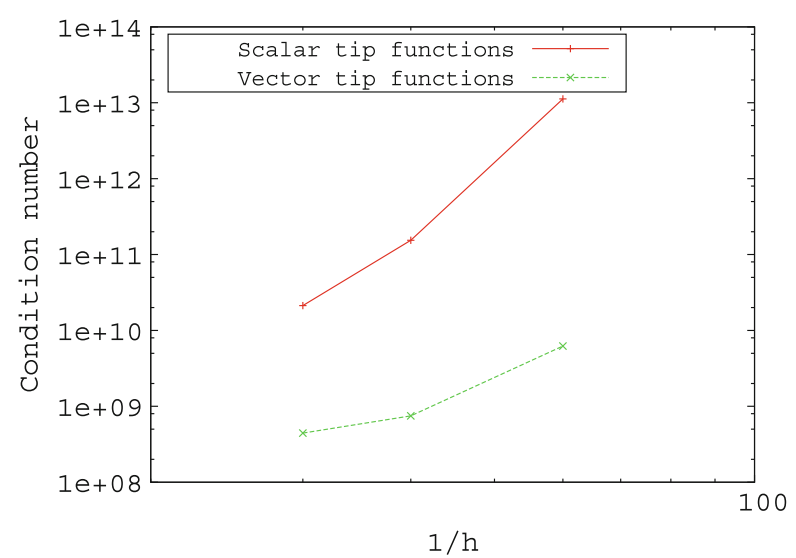

Fig. 16 The evolution of the condition number as a function of mesh size $(h)$

Finally the condition number of the stiffness matrix, defined by the following norm \|\| , is expressed as a function of element size $(h)$ in Fig. 16.

$$
\|A\|=\max _{j} \sum_{i}\left|A_{i j}\right|
$$

As shown in [6], The vector enrichment functions improve considerably the conditioning compared to their scalar counterparts. In this case, despite the difference in conditioning, the two types of tip enrichment functions lead to the same overall results. However, this improvement can be essential for more complex problems, e.g. unilateral contact, multi-crack problem, etc.

\section{Conclusions}

In the present study, the Lagrangian method is applied to consider crack lip contact in an X-FEM context. Following conclusions are drawn from the study:

- The algorithm introduced in [3], which leads to a $P 1 / P 1^{-}$formulation, is adapted in the present study by imposing a $P 0$ interpolation in the element containing the crack tip to construct the Lagrangian multiplier space along the crack surface.

- In addition to the Heaviside function, tip enrichment functions are included in the displacement discretization. We show theoretically and numerically that the discretization with both scalar and vector tip enrichment functions respects the inf-sup condition.

- The proposed method leads to a precise calculation of Lagrangian multipliers. In the case of constant pressure on the contact surface, slight oscillations (less than $1 \%$ ) have been observed around the crack tip. We show evidences that this 
oscillation can be considerably reduced by increasing the integration degree. In the case of variable pressure on the contact surface, the vector and scalar enrichment functions result in the same overall results. The application of the vector functions improves considerably the conditioning of the stiffness matrix compared to scalar ones.

- The calculation of SIFs is adapted in the present work to count for the crack lip contact.

The present study was conducted in a two-dimensional context using unstructured meshes, and only frictionless contact is considered. In the future, this work should be at first extended to three-dimensional structures and then to frictional contact. Besides, we observe that the calculation of the Lagrangian multiplier and the stress intensity is tightly related to the integration accuracy. The application of a more adapted integration strategy within the X-FEM framework will be another short-term perspective of this work.

Acknowledgements This work is funded by an SBO Project grant 110070: eSHM with AM of the Agency for Innovation by Science and Technology (IWT). The authors would like to thank C. Friebel and N. Poletz for the insightful discussion about the implementation of the numerical framework.

\section{References}

1. Amdouni, S., Moakher, M., Renard, Y.: a stabilized lagrange multiplier method for the enriched finite-element approximation of contact problems of cracked elastic bodies. Comput. Methods Appl. Mech. Eng. 270, 178-200 (2014)

2. Béchet, E., Minnebo, H., Moës, N., Burgardt, B.: Improved implementation and robustness study of the X-FEM for stress analysis around cracks. Int. J. Numer. Methods Eng. 8, 1033 1056 (2005)

3. Béchet, E., Moës, N., Wohlmuth, B.: A stable Lagrangian multiplier space for stiff interface conditions within the extended finite element method. Int. J. Numer. Methods Eng. 78, 931954 (2009)

4. Brezzi, F., Bathe, K.J.: A discourse on the stability conditions for mixed finite element formulations. Comput. Methods Appl. Mech. Eng. 53, 27-57 (1990)

5. Chapelle, C., Bathe, K.J.: The inf-sup test. Compos. Struct. 47, 537-545 (1993)

6. Chevaugeon, N., Moës, N., Minnebo, H.: Improved crack tip enrichment functions and integration for crack modeling using the extended finite element method. Int. J. Multiscale Comput. Eng. 11, 597-631 (2013)

7. Duflot, M.: A study of the representation of cracks with level sets. Int. J. Numer. Methods Eng. 70, 1261-1302 (2007)

8. Ferté E., Massin, P., Moës, N.: Interface problems with quadratic X-FEM: design of a stable multiplier space and error analysis. Int. J. Numer. Methods Eng. 11, 834-870 (2014)

9. Fries, T.P.: A corrected XFEM approximation without problems in blending elements. Int. J. Numer. Methods Eng. 75, 503-532 (2008)

10. Geniaut, S., Massin, P., Moës, N.: A stable 3D contact formulation using X-FEM. Eur. J. Comput. Mech. 16, 259-275 (2007)

11. Giner, E., Tur, M., Tarancón, J.E., Fuenmayor, F.J.: Crack face contact in XFEM using a segment to segment approach. Int. J. Numer. Methods Eng. 82, 1424-1449 (2010) 
12. Gupta, V., Duarte, C.A., Babuška, I., Banerjee, U.: A stable and optimally convergent generalized FEM (SGFEM) for linear elastic fracture mechanics. Comput. Methods Appl. Mech. Eng. 266, 23-39 (2013)

13. Gupta, V., Duarte, C.A., Babuška, I., Banerjee, U.: Stable GFEM (SGFEM): improved conditioning and accuracy of GFEM/XFEM for three-dimensional fracture mechanics. Comput. Methods Appl. Mech. Eng. 289, 355-386 (2015)

14. Ji, H., Dolbow, J.E.: On strategies for enforcing interfacial constraints and evaluating jump conditions with the extended finite element method. Int. J. Numer. Methods Eng. 61, 25082535 (2004)

15. Laborde, P., Pommier, J., Renard, Y., Salaün, M.: High order extended finite element method for cracked domains. Int. J. Numer. Methods Eng. 64, 354-381 (2005)

16. Li, F.Z., Shih, C.F., Needleman, A.: A comparison of methods for calculating energy release rates. Eng. Fract. Mech. 21, 405-421 (1985)

17. Melenk, J.M., Babuška, I.: The partition of unity finite element method: basic theory and applications. Comput. Methods Appl. Mech. Eng. 1-4, 289-314 (1996)

18. Moës, N., Dolbow, J., Belytschko, T.: A finite element method for crack growth without remeshing. Int. J. Numer. Methods Eng. 1, 131-150 (1999)

19. Moës, N., Gravouil, A., Belytschko, T.: Non-planar 3D crack growth by the extended finite element and level sets - Part I: mechanical model. Int. J. Numer. Methods Eng. 53, 2549-2568 (2002)

20. Moës, N., Béchet, E., Tourbier, M.: Imposing Dirichlet boundary conditions in the extended finite element method. Int. J. Numer. Methods Eng. 67, 1641-1669 (2005)

21. Moran, B., Shih, C.F.: Crack tip and associated domain integrals from momentum and energy balance. Eng. Fract. Mech. 27, 615-641 (1987)

22. Moran, B., Shih, C.F.: A general treatment of crack tip contour integrals. Int. J. Fract. 35, 295-310 (1987)

23. Pereira, J.P., Duarte, C.A., Jiao, X.: Three-dimensional crack growth with hp-generalized finite element and face offsetting methods. Comput. Mech. 46, 431-453 (2010)

24. Shih, C.F., Moran, B., Nakamura, T.: Energy release rate along a three-dimensional crack front in a thermally stressed body. Int. J. Fract. 30, 79-102 (1986)

25. Siavelis, M., Guiton, M.L.E., Massin, P., Moës, N.: Large sliding contact along branched discontinuities with X-FEM. Comput. Mech. 1, 201-219 (2012)

26. Stolarska, M., Chopp, D.L., Moës, N., Belyschko T.: Modelling crack growth by level sets in the extended finite element method. Int. J. Numer. Methods Eng. 51, 943-960 (2001)

27. Wyart, E.: Three-dimensional crack analysis in aeronautical structures using the substructured finite element/extended finite element method. Ph.D Thesis, Universié catholique de Louvain Faculté des Sciences Appliquées (2007)

28. Yau, J.F., Wang, S.S., Corten, H.T.: A mixed-mode crack analysis of isotropic solids using conservation laws of elasticity. J. Appl. Mech. 47, 335-341 (1980) 